\title{
Data forwarding algorithm over lossy links in wireless sensor networks
}

\author{
Tran Dang Hoa ${ }^{1}$ and Dong-Seong Kim ${ }^{2 a)}$ \\ ${ }^{1}$ Dept. of IT Convergence Engineering, School of Electronic Engineering \\ Kumoh National Institute of Technology, Gumi, South Korea \\ ${ }^{2}$ School of Electronic Engineering
}

Kumoh National Institute of Technology, Gumi, South Korea

a)dskim@kumoh.ac.kr

\begin{abstract}
This paper proposes a new forwarding algorithm over lossy wireless link that increases network performance in terms of received traffic at the sink $\left(R_{0}\right.$ packets/s) and network lifetime in wireless sensor networks (WSNs). Due to lossy wireless links, each sensor node forms around itself three different communication regions, each characterized by different packet reception rates (PRRs). The results of simulations indicate that, the proposed FOLLA (Data forwarding with lossy link awareness) algorithm outperforms the conventional algorithms with respect to $R_{0}$ and network lifetime.
\end{abstract}

Keywords: lossy wireless link, network lifetime

Classification: Network

\section{References}

[1] J. Al-Karaki, et al., "Routing techniques in wireless sensor networks: a survey," IEEE Wireless Commun., vol. 11, no. 6, pp. 6-28, Dec. 2004.

[2] Zuniga, et al., "Analyzing the transitional region in low power wireless links," Proc. 1st IEEE Communications Society Conference on Sensor and Ad Hoc Communications and Networks, pp. 517-526, Oct. 2004.

[3] J. Heo, et al., "EARQ: Energy Aware Routing for Real-Time and Reliable Communication in Wireless Industrial Sensor Networks," IEEE Transactions on Industrial Informatics, vol. 5, no. 1, pp. 3-11, Feb. 2009.

[4] T. Shu and M. Krunz, "Coverage-Time Optimization for Clustered Wireless Sensor Networks: A Power-Balancing Approach," IEEE/ACM Trans. Netw., vol. 18, no. 1, pp. 202-215, Feb. 2010.

[5] W. Heinzelman, et al., "An application-specific protocol architecture for wireless microsensor networks," IEEE Trans. Wireless Commun., vol. 1, no. 4, pp. 660-670, Oct. 2012.

[6] J. Wang, et al., "A Distance-Based Energy Aware Routing Algorithm for Wireless Sensor Networks," Sensors, vol. 10, no. 10, pp. 9493-9511, Oct. 2010.

[7] J. Deng, "Multihop/Direct Forwarding (MDF) for static wireless sensor networks," ACM Trans. Sensor Networks, vol. 5, no. 35, pp. 1-25, Nov. 2009. 


\section{Introduction}

Among data forwarding schemes of WSNs, the direct forwarding (DF) method outperforms the multi-hop forwarding (MF) method in terms of energy efficiency, but only in small-scale networks. The reason is that, sending data directly to the common sink rapidly depletes energy reserves of those nodes farthest away from it, giving rise to the hot pots [1].

In this work, data processing techniques (encoding, decoding, etc.) are applied to the lossy wireless links [2] in order to develop a mathematical model that encompasses interference, noise and PRR metrics. The lossy nature of wireless links in multi-path fading environments causes the transmission region of sending nodes is divided into three different regions: the connected region, the disconnected region and the transitional region Fig. 1. In the connected region, nodes receive almost data sent from the sending nodes (PRR approximates 1 in this region).

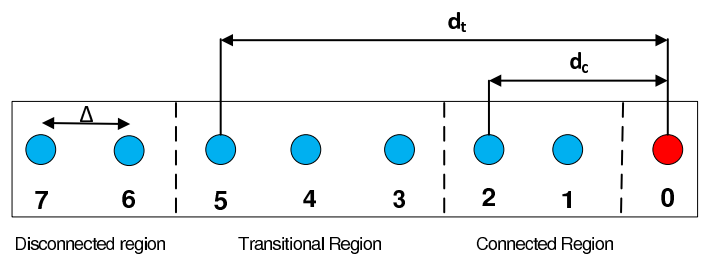

Fig. 1. Different ranges of wireless networks in terms of PRR.

The metric "HD.PRR" (the product of hop distance (HD) and PRR) in this paper is derived from solving for the LPP with the objective of maximizing $R_{0}$ and increasing the lifetime of WSNs. The process of solving the LPP is similar to the actions carried out by nodes in splitting their traffic and choosing their relay nodes in order to obtain an optimal algorithm. Based on the observations of node behaviors, a new forwarding algorithm called FOLLA is built. Using the proposed algorithm, the nodes are made aware of lossy links and they can adaptively transmit their traffics either to relay nodes with high values of "HD.PRR" or directly to the sink depending on the distance between the nodes and the sink. The FOLLA proves its simplicity and sub-optimality as comparing to the optimal algorithm.

\section{System Model}

\subsection{Network Model}

For simplicity, the proposed scheme considers a straight-line network model. However the random deployed networks can be approximated as linear networks as in [3] and [4]. In this model, the set of sensor nodes is labeled using a set of integers $S=\{0,1, . ., N\}$. Nodes are evenly distributed in a sequence from the sink (labeled 0) to the final node N. The distance between two successive nodes is a constant, $\Delta(\mathrm{m})$. The distance between any two nodes $i$ and $j$ is denoted as $d_{i j}(\mathrm{~m})$ and so $d_{i j}=\Delta(|j-i|)$. We assume that the data generation rate of each node is the same $(\lambda \operatorname{packet}(\mathrm{s}) / \mathrm{s})$. 


\subsection{Energy Model}

A transmitting energy consumption from node $i$ to $j$ with the transmitted power $P_{t x, i j}=P_{\text {elec }}+P_{t x}\left(d_{i j}\right)$ can be extended from the model in [5] as follows:

$$
E_{t x}\left(d_{i j}\right)=\frac{1}{B R}\left(P_{\text {elec }}+P_{t x}\left(d_{i j}\right)\right),
$$

where $P_{\text {elec }}$ is the power consumed by the transmitter circuits, which is a constant and $B R$ is the bit rate (bits per second). The term $P_{t x}\left(d_{i j}\right)$ is the power consumed by the power amplifier circuit at node $i$ separated from node $j$ by distance $d_{i j} . P_{t x, i j}$ is variable depending on the transmission distance $d_{i j}$ and the packet loss rate objective. For a given receiver circuit, the per bit average receiving energy consumption at receive power $P_{r x}$ is fixed, calculated by $E_{r x}=P_{r x} / B R$, where $P_{r x}=P_{\text {elec }}$.

\subsection{Lossy Wireless Link Model}

The proposed scheme adopts the lossy link model derived. The PRR at a receiving node $j$ at a distance $d_{i j}$ from a sending node $i$ is calculated as follows:

$$
\operatorname{PRR}\left(d_{i j}\right)=\left(1-\frac{1}{2} \exp \left(-\frac{\gamma\left(d_{i j}\right)}{2} \frac{1}{0.64}\right)\right)^{\rho 8 l} .
$$

The PRR values depend on parameters, such as $\gamma$ (SNR), $\rho$ (encoding ratio), $l$ (frame length in bytes), and the modulation and encoding radio schemes [2].

\subsection{Optimal Forwarding Algorithm from LPP}

In this section, we formulate the forwarding problem and build a framework for the LPP (based on the network models described in Sections 2.1 to 2.3) for which the solution is an optimal algorithm. Denoting $N_{c}$ and $N_{t}$ as the maximum numbers of hops in the connected and transitional regions, respectively,

$$
\left\{\begin{array}{l}
N_{c}=\max \left\{1,\left\lfloor\frac{d_{c}}{\Delta}\right\rfloor\right\}, \\
N_{t}=\left\lfloor\frac{d_{t}}{\Delta}\right\rfloor
\end{array}\right.
$$

where $d_{c}$ and $d_{t}$ are the radius of the connected and transitional regions, respectively.

Denoting $T_{i}$ and $R_{i}$ as data (packets/s) that node $i$ transmits and receives at an instance in time, $T_{i j}$ as the data sent to node $j$ from node $i, R_{i j}$ as the data received at node $j$ from node $i$, then we have $R_{i j}=P R R\left(d_{i j}\right) T_{i j}$. The LPP is formulated as follows:

$$
\max _{T_{i 0}, P R R\left(d_{i 0}\right)} R_{0}=\sum_{i=1}^{N}\left[T_{i 0} P R R\left(d_{i 0}\right)\right]
$$

subject to

$$
\begin{aligned}
& T_{i 0}+\sum_{j=1}^{i-1} T_{i j}=\lambda+\sum_{k=i+1}^{N}\left[T_{k i} \operatorname{PRR}\left(d_{k i}\right)\right], \\
& E_{N}=E_{i}=E_{1}, i=2, \ldots,(N-1), \\
& T_{i j} \geq 0,0 \leq \operatorname{PRR}\left(d_{i j}\right) \leq 1
\end{aligned}
$$


where the objective function (4) is to maximize the total received traffic $R_{0}$ at the sink. The constraint (5) indicates the conservation law of traffic going into and going out of node $i$. The energy consumption balance constraint is described in Equation (6), where,

$$
\begin{aligned}
& E_{i}=E_{r x} \sum_{k=i+1}^{N}\left(T_{k i} P R R\left(d_{k i}\right)\right)+\sum_{j=0}^{i-1}\left(T_{i j} E_{t x}\left(d_{i j}\right)\right) \\
& E_{N}=\sum_{j=0}^{N-1}\left(T_{N j} E_{t x}\left(d_{N j}\right)\right) \\
& E_{1}=E_{r x} \sum_{j=2}^{N}\left(T_{j 1} \operatorname{PRR}\left(d_{j 1}\right)\right)+E_{t x}(\Delta)\left[\lambda+\sum_{j=1}^{N}\left(T_{j 1} P R R\left(d_{j 1}\right)\right)\right] .
\end{aligned}
$$

$\operatorname{PRR}\left(d_{i 0}\right)$ depends on the distance between node $i$ and sink.

$$
\operatorname{PRR}\left(d_{i 0}\right)=\left\{\begin{array}{ll}
1 & \text { if } i \leq N_{c} \\
p \in(0,1) & \text { from }(2) \text { if } N_{c}<i \leq N_{t} . \\
0 & \text { if } N_{t}<i \leq N
\end{array} .\right.
$$

\section{FOLLA Algorithm}

Solving the LPP gives the optimal forwarding algorithm for network performance. The traffic splitting and forwarding rule for the nodes is presented by the set of $T_{i j}$ where $0 \leq i, j \leq N$ such that $R_{0}$ at the sink is maximized and energy consumption of the nodes is balanced.

In large-scale networks, finding the solution for the LPP can be difficult due to the complexity of the computation. In WSNs, the computations consume a considerable energy in the nodes. For such networks, the optimal forwarding method cannot be applied. Fortunately, the optimal solutions for LPP indicate trends for each node in relation to the splitting and forwarding of its data to the sink. By analyzing these trends, simpler algorithm can be formulated as near-optimal algorithm.

The energy consumed by a sending node and the PRR value at the corresponding receiving node depend on the hop distance (HD) between the nodes. For obtaining high expected PRR in networks, utilizing small distance hop seems to make sense. However, such a scheme would result in an increase in the number of hops required to reach the sink and in a corresponding increase in the energy consumed by the nodes. On the other hand, choosing larger HDs results in low PRR values and a corresponding decrease in network performance. Therefore, a trade-off between energy efficiency and network performance is required. The solution of the LPP indicates that each node tends to choose a node with the highest "HD.PRR" as its relay node. This metric is aware of lossy wireless link characteristics (i.e., different PRR values at different HDs) for forwarding data effectively.

Different applications may have different requirements on the network topology. The proposed algorithm considers a datacentric application in which there is a madatory requirement on network lifetime, and the network 


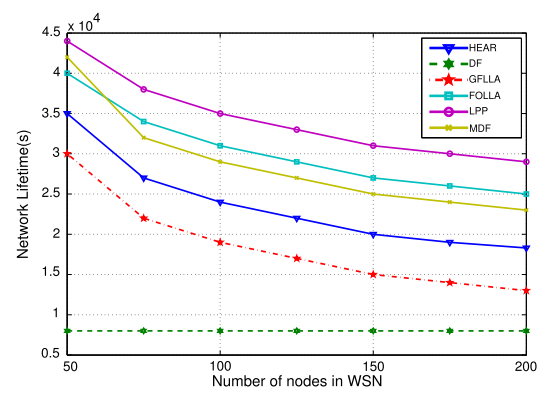

(a) Lifetime of network

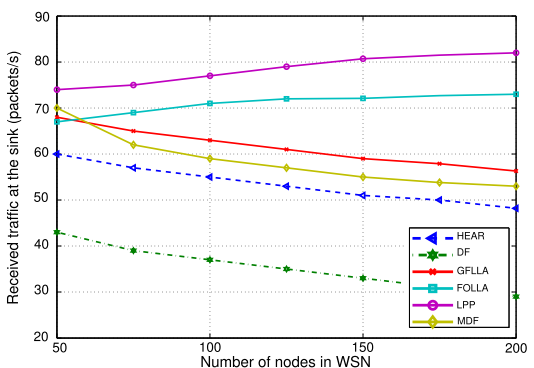

(b) Received traffic at the sink

Fig. 2. Performance evaluation with respect to related algorithms.

energy should be minimized. In other word, if we sort the nodes in routing table according to the "HD-PRR" metric (the highest metric, the better candidate), how much energy are we saving and optimal balance of consumption energy and network lifetime by choosing the best candidate.

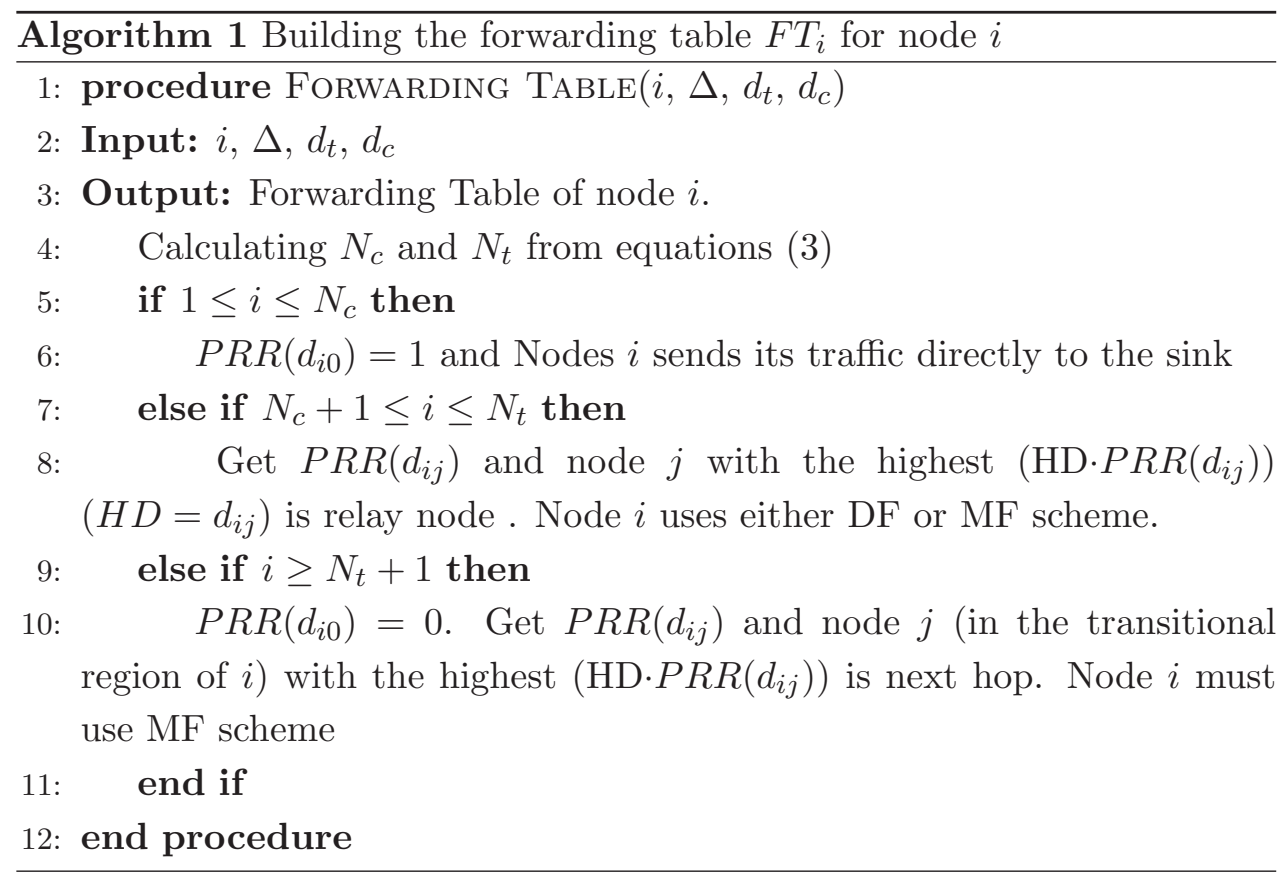

\section{Simulation Results}

In this section, the simulation results are shown and compared when the proposed algorithm is deployed in a chain networks. The initial energy of each node was set at $1 \mathrm{~J} ; \lambda=1$ packet $/ \mathrm{s}, \Delta=2 \mathrm{~m}$.

The FOLLA algorithms is compared and evaluated against several conventional forwarding algorithms. The greedy forwarding with lossy link awareness (GFLLA) algorithm is simulated and compared to analyze the effects of wireless lossy links on the network performance. The MF scheme in which each node greedily chooses the most distant node in its connected 
region to be its relay node ( $N_{c}$ hops) is used in the GFLLA algorithm. In Figure $2 \mathrm{a}$, the network lifetime ${ }^{1}$ of various forwarding algorithms is depicted. The LPP algorithm shows its optimality with the highest network lifetime.

On the other hand, with the possibility of balancing the energy of nodes with the optimal hop (eight hops in this paper's network model), HEAR algorithm in [6] achieves higher network lifetime than DF and even GFLLA algorithms. When the number of nodes in the networks is not greater than $N_{t}$, the multihop/direct forwarding (MDF) algorithm in [7] gives a better performance than the FOLLA algorithm. In all cases, FOLLA achieves a near-optimal performance and outperforms the MDF algorithm. This result is because when $N \leq N_{t}$, no nodes are in the disconnected region toward the sink so MDF can balance energy consumption better than FOLLA.

Figure $2 \mathrm{~b}$ depicts the average values of $R_{0}$ for the networks with various algorithms deployed. From the simulation results, it is clear that the LPP's algorithm gives the best performance. The effects of lossy link are shown more clearly. Although the network lifetime of HEAR is greater than that of GFLLA, $R_{0}$ of HEAR is greater at the sink than that of GFLLA. This result is because, in the HEAR algorithm, the relay node for each node is in its transitional region (optimal hop $(=8)>N_{c}$ ). In addition, when $N_{c}=7$ hops is chosen in the connected region for forwarding, $R_{0}$ in the case of GFLLA is higher than that in the case of FOLLA. However, the difference is quite small. Ultimately, FOLLA can beat GFLLA in the long run of network.

\section{Conclusion}

This paper proposes a data forwarding algorithm FOLLA for lossy WSNs. By analyzing the solution for the LPP which gives the optimal forwarding algorithm, a new metric "HD.PRR" is derived to build the near-optimal FOLLA algorithm. Simulation results indicate that FOLLA is better than related algorithm in term of received traffic rate and network lifetime.

\section{Acknowledgments}

This research was financially supported by the Ministry of Education, Science Technology (MEST) and National Research Foundation of Korea (NRF) through the Human Resource Training Project for Regional Innovation 2013 and Basic Science Research Program (NO. 2011-0025409).

${ }^{1}$ The network lifetime is the time duration from the starting of the network to the first node is depleted. 